\title{
Bandgesteuerte Kolonoskopie
}

\begin{abstract}
Eine 46-jährige Frau berichtete über Juckreiz in der Analregion, der seit drei Tagen bestand. Sie glaubte außerdem, weißliche bandartige Fremdkörper im Stuhl entdeckt zu haben.
\end{abstract}

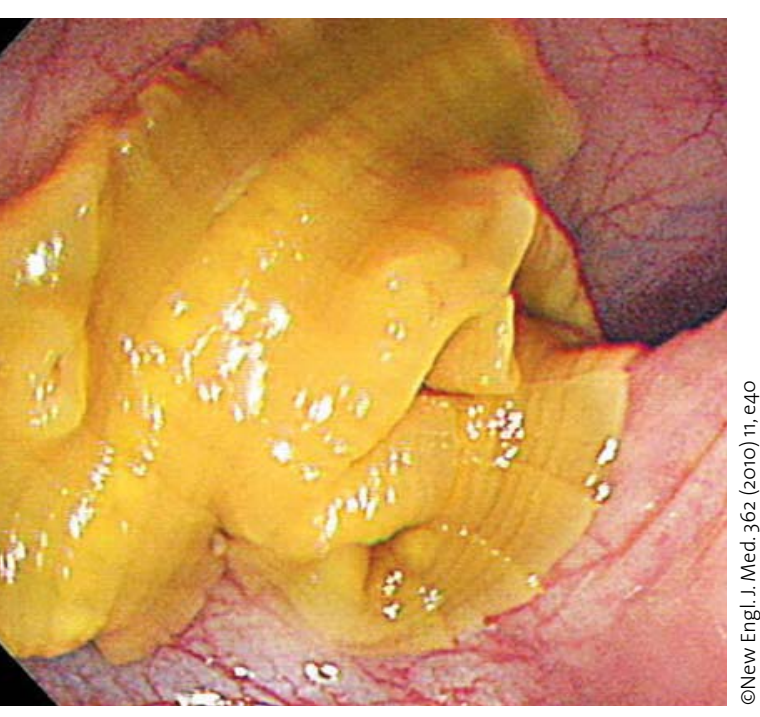

Fischbandwurm im Kolon.
- Im vergangenen Jahr hatte sie immer wieder einmal kolikartige Bauchschmerzen und vorübergehend auftretende weiche Stühle, was vom vorbehandelnden Arzt als Colon irritabile interpretiert wurde. Alle Laboruntersuchungen waren unauffällig, insbesondere lag keine Anämie vor.

Die Kolonoskopie muss sich einfach gestaltet haben, da sich der Untersucher am weißen beweglichen Band eines Fischbandwurms (Diphyllobothrium latum) orientieren konnte, dessen Kopf im terminalen Ileum festgesaugt war und dessen Schwanz sich bis in das Sigma erstreckte. Natürlich wurden der Patientin die einschlägigen Fragen gestellt, und sie erinnerte sich, dass sie etwa zwei Monate vor der Vorstellung rohe Forelle gegessen hatte. Nach einer Behandlung mit einer einzigen Dosis von Praziquantel verschwanden mit dem Abgang des Bandwurms auch die Bauchschmerzen.

\section{Kommentar}

Nimmt man eine Länge des Kolons von etwa einem Meter an, so muss es sich in diesem Fall um ein Jungtier gehandelt haben, da Fischbandwürmer zu den größten Vertretern der Bandwürmer gehören und eine Länge bis zu 20 Metern erreichen können. Im letzten Segment werden die Eier produziert und über eine Öffnung ausgeschieden. Da meistens nur ein Wurm in einem Menschen vorkommt, wird der Befall häufig nicht bemerkt. Nur wenige Patienten entwickeln den in Lehrbüchern beschriebenen Mangel an Vitamin B 12. Ungewöhnlich an dem Fall ist, dass die Patientin offensichtlich keine Eosinophilie im Blutbild aufwies.

H. S. FÜEßL =

\footnotetext{
- J. Hak Kim, J. Ho Lee

(Dongguk University College of Medicine Goyang, South Korea, e-mail: jhleemd@duih. org): Diphyllobothrium latum during colonoscopy. New Engl. J. Med. 362 (2010) 11, e40
}

\section{Wie sehr Anabolika dem Herzmuskel schaden}

\author{
Weltweit nutzen insbesondere \\ Bodybuilder verbotene anabole, \\ androgene Steroide (AAS). Trotz \\ dieser großen Verbreitung ist wenig \\ über deren kardiale Langzeiteffekte \\ bekannt.
}

- Wissenschaftler am Massachusetts General Hospital in Boston (USA) verglichen in einer kleinen Pilotstudie die Herzleistungen von 17 Gewichthebern, von denen zwölf seit mehreren Jahren regelmäßig AAS konsumierten.

Bis auf den Anabolikakonsum entsprachen die Testpersonen bezüglich Geschlecht und Alter (männlich, durchschnittlich 40 Jahre alt), Trainingsumfang und sportlicher Erfahrung, Statur und allgemeinen Gesundheitsrisiken den sieben Kontrollen. Die Steroidanwender hatten erwartungsgemäß mehr
Muskelmasse. Die systolische Leistung der linken Kammer (LV) wurde mit der Echokardiografie als LV-Ejektionsfraktion ermittelt. Auch die Leistungen während der Diastole wurden gemessen.

Die Herzstrukturen beider Gruppen waren nicht verschieden. Die AASKonsumenten hatten aber mit 50,6\% $(48,4-53,4)$ versus $59,1 \%(58,0-81,7)$ eine signifikant $(\mathrm{p}=0,003)$ kleinere LV-Ejektionsfraktion. Zehn der zwölf AAS-Nutzer hatten LV-Ejektionsfraktionen unter dem Normlimit ( $\leq 55 \%)$.

\section{Kommentar}

Mit dieser kleinen, aber eindeutigen Pilotstudie wird zum ersten Mal deutlich, dass die üblichen muskelaufbauenden Anabolika bei permanenter Anwendung die Schlagfunktionen der linken Herzkammer so schwer schädigen können, dass eine Herzinsuffizienz droht. Nicht klar ist, wie lange die Drogen eingenommen werden müssen, bis es zu diesem Schaden kommt. Natürlich muss die Studie fortgesetzt werden, um die Resultate zu bestätigen und abzusichern.

K. MALBerg = 